\title{
The benefits and risks of PrEP and kidney function
}

(title $<60$ characters including spaces)

Amanda Mocroft ${ }^{1}$, Lene Ryom ${ }^{2}$

${ }^{1}$ Dept Infection and Population Health, University College London, London, UK. ${ }^{2}$ Dept. of Infectious diseases, CHIP, University of Copenhagen, Denmark.

In the recent Lancet HIV report from Gandhi et al (ref), a modest decrease in renal function as estimated by creatinine clearance $(\mathrm{CrCl})$ in persons starting Pre-exposure prophylaxis for HIV (PrEP) was reported. There was a higher decline in those aged $\geq 40$ or with $\mathrm{CrCl}<90$. Further, the rate of decline was strongly associated with concentration of tenofovir in hair samples.

The clear and substantial benefit of PrEP has been shown in a number of studies ${ }^{1-3}$, with estimates of approximately $80 \%$ reductions in HIV-infections among those using PrEP ${ }^{3}$. Tenofovir disoproxil fumarate (TDF) and emtricitabine are currently the only recommended combination due to lack of data on other combinations ${ }^{4}$. Evidence on the use of tenofovir-alafenamide (TAF) as part of PrEP is currently limited, but results from HIV-positive persons suggest a more favourable renal profile ${ }^{16}$, which may make it an alternative for those at highest risk of renal disease. TDF has been reported to be associated with renal toxicities in HIV-positive persons enrolled in cohort studies, with increasing risk of chronic kidney disease as exposure to TDF increases ${ }^{5-7}$. This result was not evident in clinical trials demonstrating clinical efficacy of TDF in HIV-positive persons, many of which demonstrated an initial decline in estimated glomerular filtration rate associated with TDF limited to the first few months of treatment ${ }^{8 ; 9}$. The long term relationship between TDF and chronic kidney disease has only become evident when large heterogeneous cohorts of HIV-positive persons have been followed for many years.

There are a number of important caveats to note with the important results from Gandhi et al (ref). The persons included were from iPrEx open label extension of 3 clinical trials. As the authors note, persons included in clinical trials tend to be less representative of a 'normal' population ${ }^{10 ; 11}$, and typically have less underlying comorbidities, such as diabetes, hypertension, hepatitis $\mathrm{C}$ coinfection or other risk factors which would place them at a higher risk of renal disease ${ }^{12 ; 13}$. As such, although the changes in renal function are small, they may be larger when PrEP is rolled out to a more heterogeneous population. Balanced against this is the fact that although statistically significant decreases in $\mathrm{CrCl}$ were observed, whether these are clinically relevant and the extent to which they are reversible in all groups or only in those at lowest risk of chronic kidney disease when PrEP is stopped remain to be seen. Early evidence would suggest that the majority of persons with a decrease in $\mathrm{CrCl}<60$ recover their renal function on stopping $\operatorname{PrEP}^{14 ; 15}$, but as yet, this is a small group of individuals and further data is needed, as well as many years of follow-up to understand the long term clinical consequences of the changes in a laboratory based parameter.

One simple way to assess the risk and benefit of treatment is the number needed to benefit or harm. Data from Molina et al ${ }^{3}$ suggest the number needed to treat to benefit (no HIV-infection) was 18 , and the number needed to treat to harm (grade $2+$ proteinuria) was 143 . Although the benefit clearly outweighs the harm, it is worth emphasising that the number needed to treat to harm will be 
significantly lower in those aged $>40$, or with poorer renal function at starting PrEP, as illustrated by Gandhi et al (ref), where the chance of a single $\mathrm{CrCl}<70$ was significantly higher. Clearly other measures of harm which are clinically relevant are needed in this population, as well as the mean to identify those at greatest risk of harm with long term exposure to PrEP.

TDF-based PrEP can clearly benefit the majority of people who are treated. The study from Gandhi et al (ref) illustrates the importance of administering PrEP in a setting with access to regular clinical follow-up, including monitoring of renal function, until further data is available. There is an ongoing need to understand the risk of declining renal function in persons using PrEP with underlying comorbidities and higher risk of renal problems, as well as the extent to which these resolve when PrEP is stopped. This requires long term follow-up of persons exposed to PrEP, as well as exposure among more heterogeneous populations at risk of HIV acquisition.

Reference List

(1) Grant RM, Lama JR, Anderson PL, McMahan V, Liu AY, Vargas L et al. Preexposure chemoprophylaxis for HIV prevention in men who have sex with men. N Engl J Med 2010; 363(27):2587-2599.

(2) McCormack S, Dunn DT, Desai M, Dolling DI, Gafos M, Gilson R et al. Pre-exposure prophylaxis to prevent the acquisition of HIV-1 infection (PROUD): effectiveness results from the pilot phase of a pragmatic open-label randomised trial. Lancet 2016; 387(10013):53-60.

(3) Molina JM, Capitant C, Spire B, Pialoux G, Cotte L, Charreau I et al. On-Demand Preexposure Prophylaxis in Men at High Risk for HIV-1 Infection. N Engl J Med 2015; 373(23):2237-2246.

(4) EACS European AIDS Clinical Society Guidelines. Version 8.0 September 2015. http://www eacsociety org/guidelines/eacs-guidelines/eacs-guidelines html [ 2016 [cited 2016 Aug. 2]; Available from: URL:http://www.eacsociety.org/guidelines/eacs-guidelines/eacsguidelines.html

(5) Scherzer R, Estrella M, Li Y, Choi Al, Deeks SG, Grunfeld C et al. Association of tenofovir exposure with kidney disease risk in HIV infection. AIDS 2012; 26(7):867-875.

(6) Ryom L, Mocroft A, Kirk O, Worm SW, Kamara DA, Reiss P et al. Association Between Antiretroviral Exposure and Renal Impairment Among HIV-Positive Persons With Normal Baseline Renal Function: the D:A:D Studya. J Infect Dis 2013; 207(9):1359-1369.

(7) Mocroft A, Lundgren JD, Ross M, Fux CA, Reiss P, Moranne $O$ et al. Cumulative and current exposure to potentially nephrotoxic antiretrovirals and development of chronic kidney disease in HIV-positive individuals with a normal baseline estimated glomerular filtration rate: a prospective international cohort study. Lancet HIV 2016; 3(1):e23-e32.

(8) Gallant JE, Staszewski S, Pozniak AL, Dejesus E, Suleiman JM, Miller MD et al. Efficacy and safety of tenofovir DF vs stavudine in combination therapy in antiretroviral-naive patients: a 3-year randomized trial. JAMA 2004; 292(2):191-201. 
(9) Gallant JE, Parish MA, Keruly JC, Moore RD. Changes in renal function associated with tenofovir disoproxil fumarate treatment, compared with nucleoside reverse-transcriptase inhibitor treatment. Clin Infect Dis 2005; 40(8):1194-1198.

(10) Lovato LC, Hill K, Hertert S, Hunninghake DB, Probstfield JL. Recruitment for controlled clinical trials: literature summary and annotated bibliography. Control Clin Trials 1997; 18(4):328-352.

(11) Joseph G, Dohan D. Diversity of participants in clinical trials in an academic medical center: the role of the 'Good Study Patient?'. Cancer 2009; 115(3):608-615.

(12) de Boer IH. Chronic kidney disease-a challenge for all ages. JAMA 2012; 308(22):2401-2402.

(13) Jotwani V, Li Y, Grunfeld C, Choi Al, Shlipak MG. Risk factors for ESRD in HIV-infected individuals: traditional and HIV-related factors. Am J Kidney Dis 2012; 59(5):628-635.

(14) Baeten JM, Donnell D, Ndase P, Mugo NR, Campbell JD, Wangisi J et al. Antiretroviral prophylaxis for HIV prevention in heterosexual men and women. N Engl J Med 2012; 367(5):399-410.

(15) Mugwanya KK, Wyatt C, Celum C, Donnell D, Kiarie J, Ronald A et al. Reversibility of Glomerular Renal Function Decline in HIV-Uninfected Men and Women Discontinuing Emtricitabine-Tenofovir Disoproxil Fumarate Pre-Exposure Prophylaxis. J Acquir Immune Defic Syndr 2016; 71(4):374-380.

(16) Sax PE, Wohl D, Yin MT, Post F, Dejesus E, Saag M et al. Tenofovir alafenamide versus tenofovir disoproxil fumarate, coformulated with elvitegravir, cobicistat, and emtricitabine, for initial treatment of HIV-1 infection: two randomised, double-blind, phase 3, noninferiority trials. Lancet 2015; 385(9987):2606-2615. 